\title{
Appel ouvert à contributions : pandémie de COVID-19
}

\author{
Avec un processus de publication rapide
}

Diffuser cet article sur Twitter

\section{Appel spécial aux pairs évaluateurs}

La Revue PSPMC est à la recherche de pairs évaluateurs ayant une expertise interdisciplinaire et disponibles pour effectuer l'évaluation rapide de manuscrits soumis à la revue dans le cadre de l'appel à contributions sur les liens entre la pandémie de COVID-19 et les domaines de la promotion de la santé et de la prévention des maladies chroniques.

Les sujets incluent, sans s'y limiter :

- $\quad$ les maladies chroniques, leurs facteurs de risque et les liens avec la COVID-19 (p. ex. le risque accru de maladie et les effets sur la santé à long terme);

- la santé mentale;

- la consommation problématique de substances;

- l'impact et les conséquences des mesures de santé publique;

- la prestation de soins de santé préventifs;

- les données probantes sur des interventions prometteuses;

- I'équité en santé.

Si vous êtes intéressé à être pair évaluateur pour la série sur la pandémie de COVID-19 de la Revue PSPMC, veuillez nous faire parvenir un courriel à PHAC.HPCDP.Journal-Revue.PSPMC.ASPC@canada.ca nous indiquant brièvement vos domaines d'expertise, rattachements et disponibilités. Vous pouvez également fournir une notice biographique, un bref curriculum vitae ou des liens vers une biographie en ligne afin de nous aider dans le processus de sélection.

Nous demandons aux évaluateurs de s'engager à compléter au moins une évaluation dans les cinq jours suivant l'acceptation de la demande d'évaluation.

La pandémie de la nouvelle maladie à coronavirus (COVID-19) a une incidence sociétale multidimensionnelle et a, d'une façon ou d'une autre, touché tous les Canadiens. De plus, d'un point de vue de santé publique, nous voyons aussi que cette pandémie et l'épidémie de maladies chroniques à croissance lente qui affecte toutes les régions du monde s'entrechoquent.

Promotion de la santé et prévention des maladies chroniques au Canada : Recherche, politiques et pratiques (la Revue PSPMC) est la revue scientifique mensuelle en ligne de la Direction générale de la promotion de la santé et de la prévention des maladies chroniques de l'Agence de la santé publique du Canada. Les rédacteurs de la revue PSPMC vous invitent par la présente à soumettre des articles de recherche quantitative et qualitative, des commentaires, des éditoriaux et des manuscrits de type « Aperçu » qui abordent les liens entre la pandémie de COVID-19 et la promotion de la santé, les maladies chroniques et l'équité en santé.

De nombreux thèmes sont pertinents dans ce domaine, notamment :

- les associations entre maladies chroniques (et leurs facteurs de risque) et risque d'infection, de maladie grave ou d'issues moins favorables

- les effets à long terme de la COVID-19 sur les survivants, en particulier les troubles de santé mentale de longue durée comme, entre autres, la dépression et l'anxiété

- l'étude des interventions en santé publique et de leur incidence ainsi que de leurs conséquences imprévues aux niveaux individuel (p. ex. santé physique et mentale, comportements sains et favorisant la santé), familial et collectif

- $\quad$ la prestation de soins de santé préventifs pendant la pandémie

- de nouvelles données scientifiques, en particulier les résultats d'études expérimentales naturelles, sur les interventions prometteuses pour améliorer la santé publique (p. ex. mesures d'éloignement physique, protection des personnes souffrant d'une affection chronique sous-jacente) ou pour atténuer l'incidence négative des interventions (p. ex. conséquences sur la santé mentale)

- l'équité en santé et les déterminants sociaux de la santé en tant que questions transversales. 
Pour assurer une pertinence à long terme de ces publications, nous nous attendons à ce que les manuscrits analysent les répercussions qu'ont leurs résultats lors de la phase de rétablissement de la crise actuelle et au-delà.

Les manuscrits seront examinés au fur et à mesure de leur réception. Les manuscrits retenus pour les étapes suivantes seront transmis à un comité éditorial spécial chargé de cette série ainsi qu'à deux pairs évaluateurs (si le type d'article le justifie).

Nous nous efforcerons de communiquer la décision éditoriale initiale concernant les manuscrits soumis dans les 15 jours ouvrables pour un article évalué par les pairs et dans les 5 jours ouvrables pour les manuscrits sans examen par les pairs. Les manuscrits acceptés seront priorisés pour publication et seront rendus disponibles en ligne, en format HTML, et indexés " avant l'impression » antérieurement à la création de la version PDF et à leur inclusion dans un numéro régulier de la revue.

Consultez notre site Internet pour toute information sur les types d'articles et sur les directives à l'intention des auteurs pour la soumission d'un article à : https://www.canada.ca/fr/sante-publique/services/rapports-publications/promotion-sante-prevention-maladies -chroniques-canada-recherche-politiques-pratiques/information-intention-auteurs.html.

Si, avant de soumettre votre article, vous avez des questions sur sa conformité ou son adéquation, veuillez les transmettre directement à l'adresse suivante : PHAC.HPCDP.Journal-Revue.PSPMC.ASPC@canada.ca.

Information pour la soumission : Veuillez mentionner cet appel à contributions dans votre lettre d'accompagnement et transmettre votre manuscrit par courriel à l'adresse PHAC.HPCDP.Journal-Revue.PSPMC.ASPC@canada.ca. Cet appel demeure valide tant qu'un nouvel avis n’a pas été publié.

Échéance pour les soumissions : Ouvert jusqu’à nouvel ordre. 